\title{
Probing cosmic-ray distribution around Cygnus OB2
}

\author{
S. Menchiari, ${ }^{a, *}$ E. Amato, ${ }^{b}$ N. Bucciantini ${ }^{b}$ and G. Morlino ${ }^{b}$ \\ ${ }^{a}$ Università degli Studi di Siena, \\ Via Roma 56 - 53100, Siena, Italy \\ ${ }^{b}$ INAF, Osservatorio Astrofisico di Arcetri, \\ L.go E. Fermi 5, Firenze, Italy \\ E-mail: menchiari2@student.unisi.it
}

Massive star clusters (MSCs) have recently received renewed attention as possible cosmic ray (CR) factories. In fact, the conversion of few \% of the cluster kinetic energy into accelerated particles can easily account for the $\gamma$-ray emission observed in coincidence with some MSCs, in a scenario where gamma-rays would result from pions produced in collisions between the relativistic hadrons and the ambient gas. In addition, the $\gamma$-ray spatial profile suggests that CRs are continuously produced during a period compatible with the typical age of a MSC. Cygnus OB2 is one of the most massive star clusters in the Milky Way, located near the Cygnus X star-forming complex. Fermi-LAT observations of the region revealed an extended gamma-ray emission (Cygnus Cocoon) possibly coming from CRs accelerated by Cygnus OB2. Recent studies claim that this extended emission is compatible with a CR distribution of freshly accelerated escaping particles. In this work, we compare the observed $\gamma$-ray emission and its radial profile with the prediction of a theoretical model where particles are accelerated at the termination shock of the cluster wind. In particular, we study the impact that different distribution of gas around the cluster have in shaping the Cocoon morphology. While the total $\gamma$-ray emission is very well reproduced, implying a maximum energy of accelerated protons of $\sim 100 \mathrm{TeV}$, the predicted radial shape is almost flat, compatible with HAWC data but not with Fermi-LAT ones.

$37^{\text {th }}$ International Cosmic Ray Conference (ICRC 2021)

July 12th - 23rd, 2021

Online - Berlin, Germany

\footnotetext{
${ }^{*}$ Presenter
} 


\section{Introduction}

Since the early 80s, massive star clusters (MSCs) have been proposed as possibles sources of galactic cosmic rays (CRs) [9]. During the last decade, different experiments have detected extended $\gamma$-ray emission in both the high-energy and very-high-energy $\gamma$-ray bands towards several MSCs, such as Cygnus OB2 [1, 3, 10], Westerlund 1 [2], Westerlund 2 [21], NGC 3603 [17], thus strengthening the evidence for their ability to accelerate CRs. It is generally thought that the engine powering up these accelerators consists of the powerful winds blown by the hundreds of massive stars inside the clusters. On average, the kinetic energy released by the winds is $\sim 10^{38}-10^{39} \mathrm{erg} / \mathrm{s}$, so the observed $\gamma$-ray luminosity from these objects can be explained assuming a constant injection rate over a few millions of years and an acceleration efficiency of $\sim 10 \%$. At present, several models of CR production have been proposed. One possibility is that acceleration is achieved inside the cluster core, where CRs are boosted by crossing multiple shocks created by the collision of stars' winds [15] or by efficient scattering by magnetic turbulence generated in such collisions [see 7, for a review of different acceleration models]. An alternative hypothesis is that acceleration could take place at the termination shock of the cluster wind, generated by the impact of the wind with the interstellar medium (ISM) [13]. Depending on the model, the shape of the CR distribution around the MSC will change, thus affecting the morphology of the observed $\gamma$-ray emission.

Cygnus OB2 is one of the most massive MSC in the Milky Way, located towards the Cygnus- $\mathrm{X}$ star-forming region at $1.4 \mathrm{kpc}$. Recently, $\gamma$-ray emission (Cygnus cocoon) showing a complex morphology has been detected in a broad energy range, from $\mathrm{GeV}$ [3] to hundreds of TeV[1]. The observed radial $\gamma$-ray flux seems to agree, under the assumption of a uniformly distributed ISM, with a distribution of escaping CRs characterized by a $1 / \mathrm{r}$ trend [5]. However, since the hadronic $\gamma$-ray emission is the result of the convolution between the ISM and the CR distributions, to better probe the CR distribution function one should take into account a more realistic ISM template based on the 3D distribution of the MCs. Here we show that the $\gamma$-ray morphology may vary a lot depending on the relative position of the MCs and the MSC. Our study also highlights the importance of the distribution of the diffuse neutral gas component when analyzing the spectral information.

In this work, we compare the observed $\gamma$-ray emission from the Cygnus cocoon, both in terms of total spectral energy distribution and spatial morphology, with the hadronic emission as predicted by particle acceleration at the wind termination shock assuming different templates for the gas distribution.

\section{Cosmic ray distribution around massive star clusters}

The interaction of the collective, powerful winds from a MSC can excavate a cavity inside the ISM. The so generated bubble can be divided into two distinct parts: an inner region, upstream of the wind termination shock, and a zone downstream of the termination shock, filled with the shocked wind material, which expands adiabatically in the ISM. Assuming for Cygnus OB2 a wind kinetic luminosity of $L_{\mathrm{w}}=2 \times 10^{38} \mathrm{erg} \mathrm{s}^{-1}$ and a mass loss ratio of $\dot{M}=10^{-4} M_{\odot} \mathrm{yr}^{-1}$, the wind speed is estimated to be $u_{1} \simeq 2500 \mathrm{~km} / \mathrm{s}$. Assuming that the system has evolved in a region with an average density of $\rho_{\mathrm{H}}=10 \mathrm{~m}_{p} \mathrm{~cm}^{-3}$, and taking into account the age of Cygnus OB2, $t_{\mathrm{age}}=3 \mathrm{Myr}$, the position of the termination shock and the forward shock of the expanding cavity can be estimated 
[20] as $R_{\mathrm{TS}}=0.7 \times L_{\mathrm{w}}^{-1 / 5} \dot{M}^{1 / 2} u_{1}^{1 / 2} \rho_{\mathrm{H}}^{-3 / 10} t_{\text {age }}^{2 / 5} \simeq 16 \mathrm{pc}$ and $R_{b}=0.76 \times\left(L_{\mathrm{w}} / \rho_{\mathrm{H}}\right)^{1 / 5} t_{\mathrm{age}}^{3 / 5} \simeq 98 \mathrm{pc}$, respectively.

We model the distribution of CRs around OB2 considering the case where acceleration takes place at the cluster wind termination shock [13]. In this model, under the assumption of a steadystate regime and spherical geometry, particles are injected and accelerated at the termination shock and then escape from the system through a combination of advection and diffusion. The CRs distribution upstream $\left(f_{1}\right)$ and downstream $\left(f_{2}\right)$ of the TS, as well as the one in the unperturbed region outside the bubble $\left(f_{\mathrm{ISM}}\right)$ are given by [13]:

$$
\begin{gathered}
f_{1}\left(r<R_{\mathrm{TS}}, E\right) \simeq f_{\mathrm{TS}}(E) \exp \left[-u_{1}\left(R_{\mathrm{TS}}-r\right) / D_{1}(E)\right] \\
f_{2}\left(R_{\mathrm{TS}}<r<R_{b}, E\right)=f_{\mathrm{TS}}(E) e^{\alpha(r)} \frac{1+\beta\left[e^{\alpha\left(R_{b}\right)} e^{-\alpha(r)}-1\right]}{1+\beta\left[e^{\alpha\left(R_{b}\right)}-1\right]}+f_{\mathrm{gal}}(E) \frac{\beta\left[e^{\alpha(r)}-1\right]}{1+\beta\left[e^{\alpha\left(R_{b}\right)}-1\right]} \\
f_{\mathrm{ISM}}\left(r>R_{b}, E\right)=f_{2}\left(R_{b}, E\right) \frac{R_{b}}{r}+f_{\mathrm{gal}}(E)\left(1-\frac{R_{b}}{r}\right)
\end{gathered}
$$

where

$$
\alpha(r)=u_{2} R_{\mathrm{TS}} \frac{\left(1-R_{\mathrm{TS}} / r\right)}{D_{2}} ; \beta(E)=\frac{D_{\mathrm{ISM}}(E) R_{\mathrm{TS}}}{u_{2} R_{b}^{2}} .
$$

The subscripts 1 and 2 refer to regions upstream and downstream of the TS, respectively. The wind speed is constant in the cold wind region, $u=u_{1}$, while in the shocked wind region is $u=u_{2}\left(r / R_{\mathrm{TS}}\right)^{-2}$, where $u_{2}=u_{1} / 4$ (for strong shocks). $D$ is the diffusion coefficient assumed to be Kolmogorov-like as a consequence of turbulence generated by the wind inhomogeneities. To estimate $D$ we assume that a fraction $\sim 5 \%$ of the wind kinetic energy is converted into turbulent magnetic field. Eq.(1) is a first order approximation to the full solution presented in [13] but is adequate for the aims of the present work.

Finally, $f_{\text {gal }}$ is the average distribution of the Galactic CR sea as inferred from AMS-02 data [4], while $f_{\mathrm{TS}}$ is the distribution of accelerated particles at the TS. The general solution for $f_{\mathrm{TS}}$ is quite involved [see 13], hence here we prefer to adopt a more simple expression given by a power law plus an exponential cut-off:

$$
f_{\mathrm{TS}}(E)=A\left(\frac{E}{1 \mathrm{TeV}}\right)^{-a} \exp \left[-\left(\frac{E}{E_{\max }}\right)^{b}\right] .
$$

where $A, a, b$ and $E_{\max }$ are parameters that we will infer from comparison with the data. Notice that the normalization $A$ is connected to the $\mathrm{CR}$ acceleration efficiency, $\epsilon_{\mathrm{CR}}$, defined as the fraction of wind kinetic luminosity converted into CRs, namely $\epsilon_{\mathrm{CR}} L_{w}=L_{\mathrm{CR}} \equiv 4 \pi R_{\mathrm{TS}}^{2} u_{2} \int f_{\mathrm{TS}}(E) E d E$.

Eqs. (1)-(3) represent a steady-state solution valid in the limit of infinite time. A more realistic approach should account for the time evolution of the system which implies that particles can propagate up to a maximum distance $R_{\text {cut }}(E)$ related to the cluster age. An approximate way to estimate such a distance is by using the energy conservation which, neglecting energy losses, gives

$$
\int_{0}^{R_{\text {cut }}(E)} 4 \pi r^{2}\left[f(r, E)-f_{\text {gal }}(E) \Theta\left(r-R_{\mathrm{TS}}\right)\right] d r=4 \pi R_{\mathrm{TS}}^{2} t_{\mathrm{age}} u_{2} f_{\mathrm{TS}}(E) .
$$

It is worth noticing that $R_{\text {cut }} \simeq R_{\mathrm{TS}}$ for $E \lesssim 1 \mathrm{TeV}$. This happens because at low energy CRs are dominated by advection and remain trapped inside the expanding bubble. At higher energies, 
instead, diffusion dominates and CRs are free to escape from the bubble, producing a distribution $\propto 1 / r$ up to $R_{\text {cut }}$ (see Fig. 1).
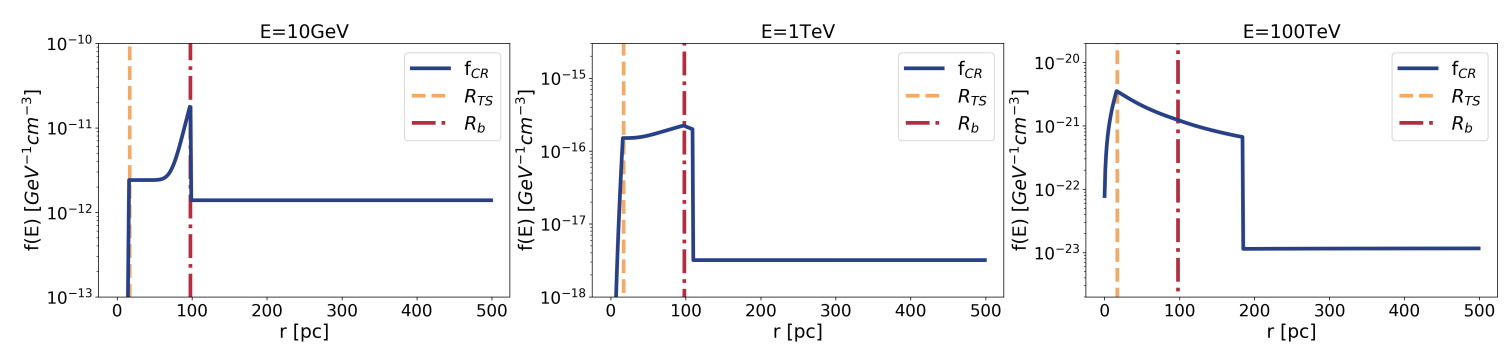

Figure 1: Radial cosmic ray distribution function around $\mathrm{OB} 2$ for three different energies: $10 \mathrm{GeV}$ (left panel), $1 \mathrm{TeV}$ (center panel) and $100 \mathrm{TeV}$ (right panel).

\section{Interstellar medium distribution}

We model the ISM as a combination of neutral $(\mathrm{HI})$ and molecular $\left(\mathrm{H}_{2}\right)$ hydrogen, considering a region centered on the Cocoon with a size of $6^{\circ} \times 6^{\circ}$ (fig 2). We use high-resolution $21 \mathrm{~cm}$ line data from the Canadian Galactic Plane survey to trace the amount of $\mathrm{HI}$ in the region. To estimate the column density, we follow the same approach adopted in [5]. For the molecular component, we use, when available, a combination of high-resolution ${ }^{13} \mathrm{CO}$ observations from the Nobeyama radio telescope [19] and low-resolution observation of ${ }^{12} \mathrm{CO}$ from the composite galactic survey of [11]. To obtain the $\mathrm{H}_{2}$ column density from the ${ }^{13} \mathrm{CO}$ and ${ }^{12} \mathrm{CO}$ tracers, we proceed as follows. For the ${ }^{12} \mathrm{CO}$, we assume a constant conversion factor $X_{\mathrm{CO}}=1.68 \cdot 10^{20}$ [3]. For the ${ }^{13} \mathrm{CO}$ we, assume local thermodynamic equilibrium, and we compute the ${ }^{13} \mathrm{CO}$ column density using the same equations of [8]. We then convert the obtained column density into $\mathrm{H}_{2}$ column density by considering a conversion ratio of $\left[\mathrm{H}_{2}\right] /\left[{ }^{13} \mathrm{CO}\right]=69 \cdot 10^{4}[6]$.

In all cases, we kinematically cut radio observations between $-20 \mathrm{~km} / \mathrm{s}$ and $20 \mathrm{~km} / \mathrm{s}$, removing in this way the contribution of both Perseus and the Outer spiral arms. Due to the kinematic ambiguity in the direction of the Cygnus region, all the material selected using this velocity range is distributed up to $4 \mathrm{kpc}$ [14], which directly translates into a large uncertainty on the MCs positions. However, it is generally believed that the majority of the observed MCs are located near Cygnus OB2, with few exceptions that may be displaced in coincidence with the foreground Cygnus Rift structure at $0.6-0.8 \mathrm{kpc}$ [18]. Indeed, measures of maser parallaxes pinpoint some of the most massive MCs in the area: DR21, DR 20 and $\mathrm{W} 75 \mathrm{~N}$ at $1.5,1.46$ and $1.3 \mathrm{kpc}$, respectively [16]. It is therefore reasonable to assume that the rest of the MCs are scattered in a few hundreds of pc around OB2.

We consider and compare two different models for the ISM distribution. In the first case, we assume a simplified situation where all the observed material is distributed uniformly along the line of sight in a range $\pm 400 \mathrm{pc}$ around the cluster position. The second model consists of a more realistic scenario where the diffuse neutral component is placed outside the cavity created by the cluster, while the MCs are positioned at a random distance from $\mathrm{OB} 2$ (in a range of $\pm 400 \mathrm{pc}$ ). We consider a total of $24 \mathrm{MCs}$ in the area (see Fig. 2), manually selecting them by taking into account both their spatial shape and their CO emission lines spectral velocities. We further assume a neutral 
density of $10^{-3} m_{p} \mathrm{~cm}^{-3}$ inside the hot bubble, corresponding to a total mass of $\simeq 300 M_{\odot}$ that is the amount of mass ejected by the winds in $3 \mathrm{Myr}$. In the free wind zone (i.e. $r<R_{\mathrm{TS}}$ ), instead, the density goes like $r^{-2}$ but the total amount of mass is negligible, being $\lesssim 1 M_{\odot}$.
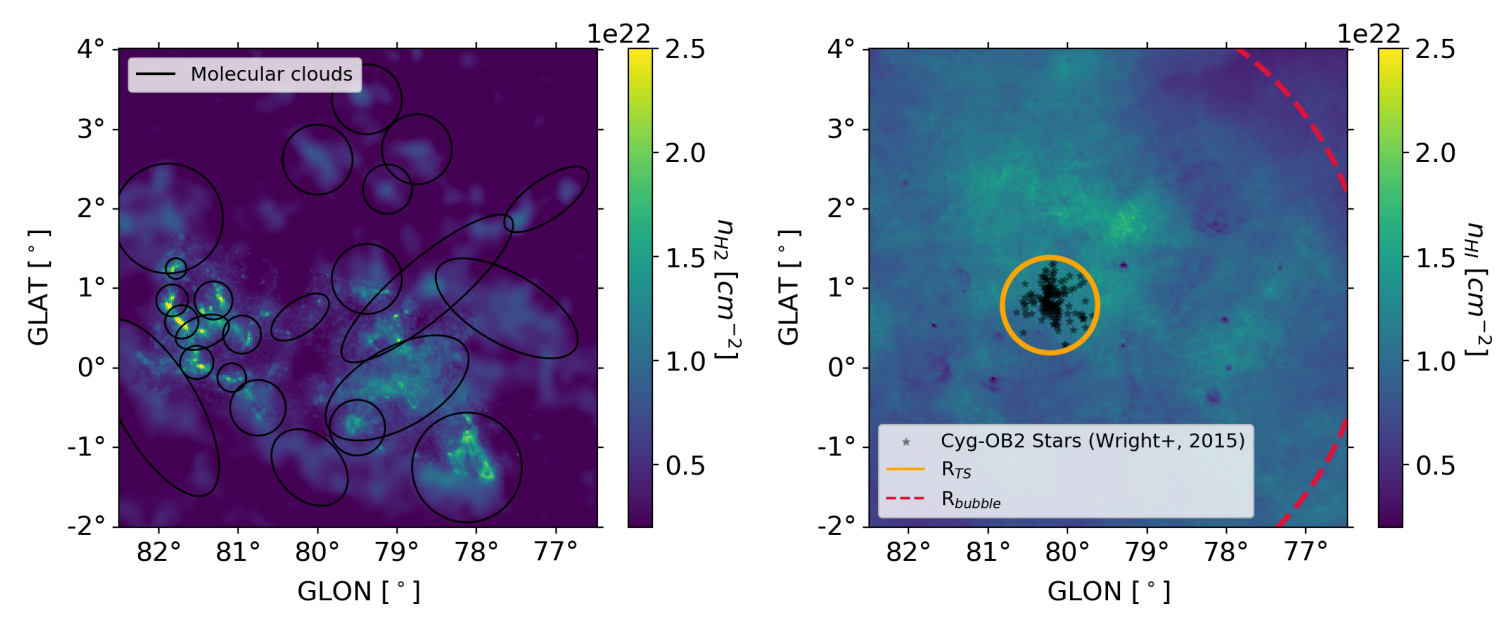

Figure 2: Column density of the molecular (left) and neutral (right) ISM components. Black circles and ellipses mark the MCs in the region used to create the complex 3D ISM template. We selected MCs using both morphology and velocity criteria. Orange and red circle on the right panel mark the termination and the forward shocks, respectively. Black stars are the position of the $\mathrm{O}$ and B stars of Cygnus OB2.

\section{Results}

Because Cygnus Cocoon is an extended source, we estimate the expected $\gamma$-ray flux from each single pixel having a size $\Omega=\left(0.02^{\circ}\right)^{2}$ and centered at Galactic coordinates $(l, b)$ by integrating the emissivity along the line of sight coordinate $z$, i.e.

$$
\phi_{\gamma}\left(l, b ; E_{\gamma}\right)=\iint \frac{c \Omega r^{2}}{4 \pi r^{2}} n_{\mathrm{gas}}(l, b, z) f_{\mathrm{CR}}\left(l, b, z ; E_{p}\right) \frac{d \sigma\left(E_{p}, E_{\gamma}\right)}{d E_{p}} d E_{p} d z
$$

where $d \sigma\left(E_{p}, E_{\gamma}\right) / d E_{p}$ is the cross-section for hadronic $\gamma$-ray production through the $\pi_{0}$ channel [12], $f_{\mathrm{CR}}\left(l, b ; E_{p}\right)$ is the distribution of CRs around Cygnus $\mathrm{OB} 2$ and $n_{\mathrm{gas}}$ is the target density. Notice that at the $\mathrm{OB} 2$ distance, $d_{\mathrm{OB} 2}=1.4 \mathrm{kpc}$, the area corresponding to the each pixel is $\Sigma \simeq 0.24 \mathrm{pc}^{2}$. As described in $\S 3$, we examine both cases of a constant distributed gas plus a more complex 3D template where MCs are placed randomly along the line of sight in such a way that the total column density for each pixel remains constant. We than construct 100 different realizations of MCs distribution to understand how the uncertainty in cloud position impact the prediction of $\gamma$-ray emission.

We than extract the spectral energy distribution (SED) from a circular region with radius $2.2^{\circ}$ centered on the OB2 cluster. The result is shown in Fig. 4a and compared with available data. Notice that observed data points in the original publication have been obtained fitting the emission with a 2D Gaussian which extends beyond our region of interest (ROI), hence we have rescaled those data to account only for the flux from our ROI. All curves are obtained using $a=2.1$, 

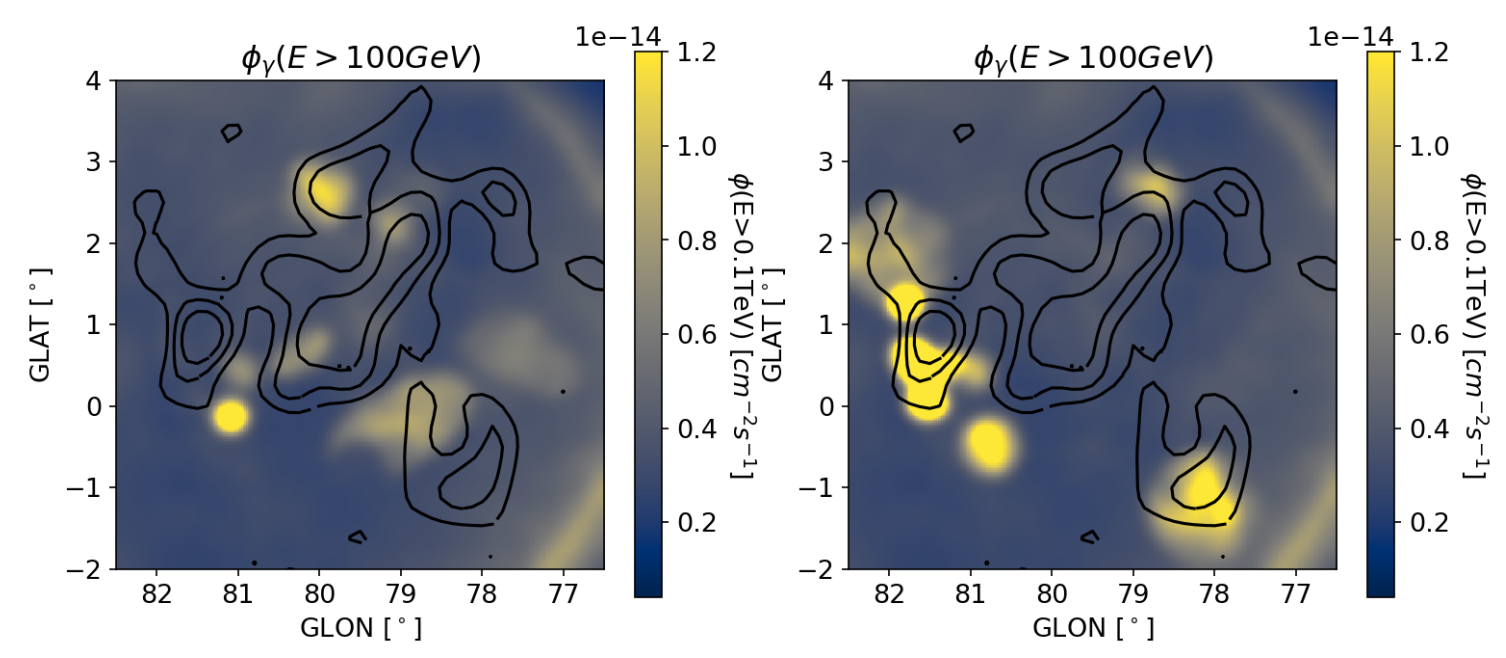

Figure 3: Observed $\gamma$-ray morphology for two different random realizations of the ISM template. Black contours represent the emission observed by Fermi. Both maps have been smoothed using a $0.1^{\circ}$ gaussian kernel.

$b=1, E_{\max }=100 \mathrm{TeV}$ in Eq. (4), while the normalization is $A=0.6 \times 10^{-16} \mathrm{~cm}^{-3} \mathrm{GeV}^{-1}$ and $A=1.64 \times 10^{-16} \mathrm{~cm}^{-3} \mathrm{GeV}^{-1}$ for model with constant ISM distribution (black dashed line) and for randomly placed MCs (grey lines). Those values correspond to an acceleration efficiency $\epsilon_{\mathrm{CR}}=1.3 \%$ and $3.6 \%$, respectively.

One can see that changing the position of MCs does not particularly affect the total $\gamma$-ray flux which varies at most by a factor of $\sim 2$ around $100 \mathrm{GeV}$. However the observed $\gamma$-ray morphology changes: Fig. 3 shown the integrated flux for $E_{\gamma}>100 \mathrm{GeV}$ for two different realizations of the ISM template compared with the contours of the Cygnus Cocoon observed by Fermi [3]. This is a direct consequence of low-energy CRs being trapped inside the cavity, which is a low-density environment filled with the mass ejected from the winds. The possible presence of MCs inside the expanding bubble introduces a large mass target for hadronic $\gamma$-ray production, therefore significantly affecting the observed morphology.

The sharp rise of the SED observed below few $\mathrm{GeV}$ is due to the fact that the emission is dominated by the background Galactic CRs, rather than by CRs coming from the stellar cluster. As so, this part of the spectrum is the most sensitive to the way the background $\gamma$-ray emission is subtracted from the data.

A very important piece of information that can help us to disentangle between different acceleration models is the CR spatial distribution that can be inferred from the spatial profile of $\gamma$-ray emission. Hence, we analyze the expected radial profile using the different ISM models by extracting the $\gamma$-ray luminosity for energies higher than $10 \mathrm{GeV}$ and $1 \mathrm{TeV}$, in 4 rings centered on OB2's position, following the method used by [5]. The obtained profiles are shown in Fig. 4b and and compared with data. Our results are compatible with HAWC and Fermi-LAT data, with the exception of the inner ring of Fermi-LAT, which show a much larger flux. Indeed this inner point has been used to infer a CR distribution $\propto 1 / r$ suggestive of a pure diffusion transport [5]. However, the region used to extract the profiles extend to a maximum radius of $\simeq 50 \mathrm{pc}$ from $\mathrm{OB} 2$, hence 


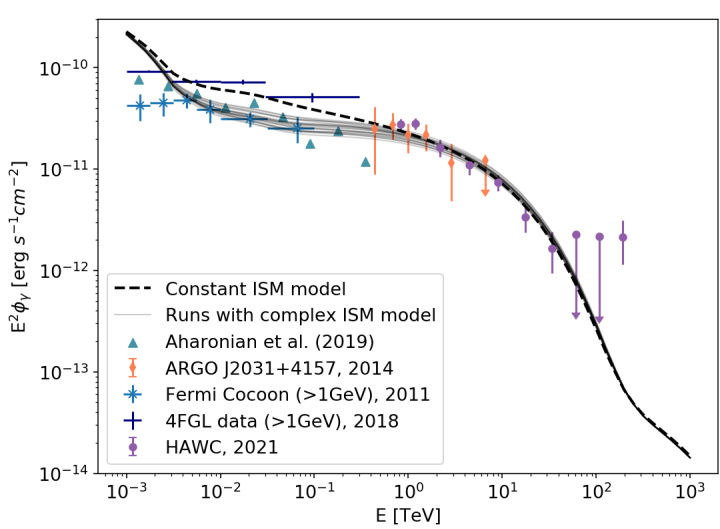

(a)

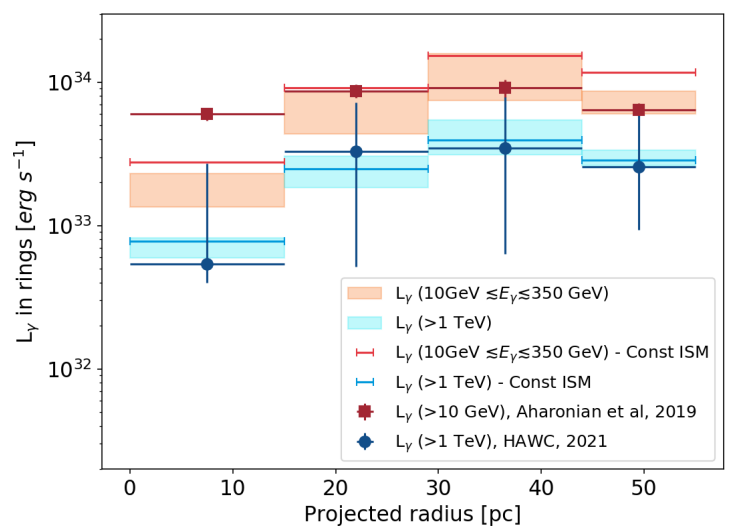

(b)

Figure 4: (a) Spectral energy distribution in the case of a constant distributed ISM along the line of sight (dashed black line) and for 100 diverse realizations of the complex ISM template with MCs distributed at randoms distance from OB2 (thin gray lines). The data points from different experiments are scaled with a factor of 2.2 for Fermi (blue cross, light blue triangle, and dark blue points), 1.9 for Argo (orange squares), 2.42 for HAWC (pink circles) to take into account only the flux coming from a region of $2.2^{\circ}$ centered on OB2 (see text). (b) $\gamma$-ray luminosity in four rings $\left(0-0.6^{\circ}, 0.6-1.2^{\circ}, 1.2-1.8^{\circ}\right.$ and $\left.1.8-2.2^{\circ}\right)$ centered on OB2. The luminosity is calculated considering $10 \mathrm{GeV} \lesssim E_{\gamma} \lesssim 350 \mathrm{GeV}$ and $E_{\gamma} \gtrsim 1 \mathrm{TeV}$, in both cases of a constant (red and blue lines) and complex 3D ISM models (red and blue boxes). The height of the boxes corresponds to the maximum observed luminosity spread for 100 different realizations of the complex ISM model.

falling inside the cavity generated by the cluster winds. In our model, the CR distribution inside this area (once integrated over the line of sight) for energies $\ll E_{\max }$ is almost flat due to the fact that transport is dominated by the advection, explaining the flatness of the $\gamma$-ray profile also at low energies.

\section{Conclusions}

In the present work, we compared the $\gamma$-ray emission expected from the distribution of CRs accelerated by Cygnus OB2, assuming that acceleration happens at the wind's termination shock, with the one observed by several experiments in the high-energy and very-high-energy bands. We computed the $\gamma$-ray emission considering both cases of ISM uniformly distributed along the line of sight and a more realistic model with MCs displaced at random distances from Cygnus OB2. The obtained SED well reproduces all the observations, and at energies of few tens of $\mathrm{GeV}$, the spread introduced by the position of molecular clouds is small. However, the distance of MCs significantly changes the observed morphology. The extracted radial luminosity profile shows an average flat profile at both low and high energies, which is compatible with HAWC data but not with Fermi observations.

In the future, we aim to consider also the case of a distribution of CRs accelerated inside the cluster by wind-wind collisions. In addition, we intend to study the contribution of leptonic emission from accelerated electrons. 


\section{References}

[1] Abeysekara, A. U., Albert, A., Alfaro, R., et al. 2021, Nature Astronomy

[2] Abramowski, A., Acero, F., Aharonian, F., et al. 2012, Astronomy \& Astrophysics, 537, A114

[3] Ackermann, M., Ajello, M., Allafort, A., et al. 2011, Science, 334, 1103

[4] Aguilar, M., Aisa, D., Alpat, B., et al. 2015, PRL, 114, 171103

[5] Aharonian, F., Yang, R., \& de Oña Wilhelmi, E. 2019, Nature Astronomy, 3, 561

[6] Bolatto, A. D., Wolfire, M., \& Leroy, A. K. 2013, Annual Review of Astronomy and Astrophysics, 51, 207, arXiv: 1301.3498

[7] Bykov, A. M., Marcowith, A., Amato, E., et al. 2020, , 216, 42

[8] Caselli, P., Walmsley, C. M., Zucconi, A., et al. 2002, The Astrophysical Journal, 565, 344, arXiv: astro-ph/0109023

[9] Cesarsky, C. J. \& Montmerle, T. 1983, Space Science Reviews, 36, 173

[10] Collaboration, T. A.-Y., Bartoli, B., Bernardini, P., et al. 2014, The Astrophysical Journal, 790, 152, arXiv: 1406.6436

[11] Dame, T. M., Hartmann, D., \& Thaddeus, P. 2001, The Astrophysical Journal, 547, 792

[12] Kafexhiu, E., Aharonian, F., Taylor, A. M., \& Vila, G. S. 2014, Physical Review D, 90, 123014, arXiv: 1406.7369

[13] Morlino, G., Blasi, P., Peretti, E., \& Cristofari, P. 2021, Monthly Notices of the Royal Astronomical Society, 504, 6096, arXiv: 2102.09217

[14] Reid, M. J., Dame, T. M., Menten, K. M., \& Brunthaler, A. 2016, The Astrophysical Journal, 823,77

[15] Reimer, A., Pohl, M., \& Reimer, O. 2006, The Astrophysical Journal, 644, 1118

[16] Rygl, K. L. J., Brunthaler, A., Sanna, A., et al. 2012, Astronomy \& Astrophysics, 539, A79

[17] Saha, L., Domínguez, A., Tibaldo, L., et al. 2020, The Astrophysical Journal, 897, 131, arXiv: 2006.00274

[18] Schneider, N., Bontemps, S., Simon, R., et al. 2006, Astronomy \& Astrophysics, 458, 855

[19] Takekoshi, T., Fujita, S., Nishimura, A., et al. 2019, The Astrophysical Journal, 883, 156, arXiv: 1907.12776

[20] Weaver, R., McCray, R., Castor, J., Shapiro, P., \& Moore, R. 1977, , 218, 377

[21] Yang, R.-z., de Oña Wilhelmi, E., \& Aharonian, F. 2018, Astronomy \& Astrophysics, 611, A77 